\title{
An optimal rebuilding strategy for a decremental tree problem
}

\author{
Nicolas Thibault, Christian Laforest \\ Tour Evry 2, LaMI/IBISC, Université d'Evry, 523 place des terrasses, 91000 EVRY \\ France $\{$ nthibaul, laforest $\} @ l a m i . u n i v-e v r y . f r$
}

\begin{abstract}
This paper is devoted to the following decremental problem. Initially, a graph and a distinguished subset of vertices, called initial group, are given. This group is connected by an initial tree. The decremental part of the input is given by an on-line sequence of withdrawals of vertices of the initial group, removed on-line one after one. The goal is to keep connected each successive group by a tree, satisfying a quality constraint: The maximum distance (called diameter) in each constructed tree must be kept in a given range compared to the best possible one. Under this quality constraint, our objective is to minimize the number of critical stages of the sequence of constructed trees. We call "critical" a stage where the current tree is rebuilt. We propose a strategy leading to at most $O(\log i)$ critical stages ( $i$ is the number of removed members). We also prove that there exist situations where $\Omega(\log i)$ critical stages are necessary to any algorithm to maintain the quality constraint. Our strategy is then worst case optimal in order of magnitude.
\end{abstract}

A lot of works have been devoted to the construction of trees spanning a given set of vertices in a graph. For example the Steiner tree problem, where the goal is to span a set (called group) of distinguished vertices (called members) with a minimum weight tree, has been extensively studied. As the problem is NPcomplete, numerous approximation algorithms have been designed (see $[1,3]$ for example). In [8], Waxman was the first author to present the on-line version of this problem in which vertices to add in, or to remove from, the current group revealed one by one (see [2] references on on-line problems). In this first paper, he divides the problem into two categories: A model in which "heavy" changes of the current tree are not allowed and a model in which changes are allowed. Then, Imase and Waxman proposed in [4] two different strategies corresponding to the two models above. In the first one the tree is just incremented or decremented and the degradation of weight is evaluated, whereas in the second one they allow changes in the current tree to maintain a guaranty on the weight. At each stage, they prove that they construct with the first strategy a tree whose weight is at a logarithmic ratio compared to the optimal one (i.e. the weight of a Steiner tree of the current group), and that they construct with the second strategy a tree whose weight is at a constant ratio compared to the optimal one. They give for the second strategy an upper bound of $O(\sqrt{i})$ on the number of elementary changes per stage (where $i$ is the number of new members). However, the tree can 
potentially be changed at each stage; this means that each stage is potentially what we call later a critical stage.

In [6], a very similar on-line Steiner tree problem with a delay constraint from one node to the others is studied. But the authors only evaluate their method with simulations, and they give no upper bound for the different competitive ratios. Note that in $[4,6]$, only the number of elementary changes is taken into account to measure the level of damage due to the allowed changes in the current tree.

In this paper we are concerned with a decremental group problem where the members to remove are revealed on-line one by one. However, we do not focus on the same objective function (the weight of the tree) but on a different measure: The diameter of the current group induced by the current tree. Note that we consider here a model in which changes are allowed because it can easily be shown that any on-line algorithm without critical stage cannot guarantee a constant competitive ratio (for the diameter objective function we consider in this paper). That is why we fix here a "relative budget", called quality constraint, on the diameter and we propose an algorithm minimizing the number of critical stages necessary to guarantee this budget constraint at each stage. Note also that we have proved that our algorithm leads to a constant number of elementary changes per stage in average (but we do not give in this paper the definitions and the proof associated to this problem because of the limitation on the number of pages).

A motivation for such model and objective function is the construction of connection structures for groups of members in networks. An important QoS parameter is latency that is expressed here in terms of maximum distance between users. This maximum distance must be guaranteed (our quality constraint). However, this must be done by minimizing the number of critical stages since they induce perturbations in communications in the current group (implying many re-routing operations between members in the current tree).

In Section 1 we describe more formally our problem. More precisely, in Subsection 1.1 we describe and motivate the constraints (namely the tree and quality constraints) that must be satisfied at each stage. In Subsection 1.2 we give the definitions of a critical stage. In Subsection 2.1 we propose an algorithm satisfying the construction constraints (in Section 2.2). In Subsection 2.3 we prove that our algorithm leads to at most $O(\log i)$ critical stages (where $i$ is the number of removed members). We prove in Section 3 that our strategy is worst case optimal in order of magnitude for the number of critical stages criterion by constructing a scenario in which at least $\Omega(\log i)$ critical stages are necessary for any algorithm to satisfy the quality constraint. These results show that our algorithm is worst case optimal for the number of critical stages.

\section{Definitions and notations}

Let $G=(V, E, w)$ be any connected weighted graph representing a network. $V$ is the set of vertices (modeling the nodes of the network), $E$ the set of edges 
(modeling the set of physical links) and $w$ a positive weight function of the edges (modeling the length of the edges). We denote by $d_{G}(u, v)$ the distance between $u$ and $v$ in $G$, i.e. the sum of the weights of the edges of a minimum weight path between $u$ and $v$ in $G$.

Definition 1 (Diameter of a group $M)$. Let $G=(V, E, w)$ be a graph and let $M \subseteq V$ be a group. We denote the diameter of $M$ in $G$ by

$$
D_{G}(M)=\max \left\{d_{G}(u, v): u, v \in M\right\}
$$

\subsection{Construction constraints}

In our problem, the graph $G=(V, E, w)$ and an initial group $M_{0} \subseteq V$ are given (with $M_{0} \neq \varnothing$ ). For example, in a meeting on network (called net-meeting) this initial group $M_{0}$ represents the set of members present at the beginning of the meeting. A structure, noted $T_{0}=\left(V_{0}, E_{0}\right)$, must be created to connect the members of $M_{0}$ ( $T_{0}$ spans $M_{0}$ in $G: M_{0} \subseteq V_{0} \subseteq V, E_{0} \subseteq E$ ).

However, members may leave the meeting. These members must be removed from the current group (we underline that they are not removed from the underlying graph $G$ ). Let $m_{0}=\left|M_{0}\right|$ be the size of the initial group. Let $u_{1}, u_{2}, \ldots, u_{i}, \ldots\left(i \leq m_{0}-1\right)$ be the sequence of members to remove. For every $i, 1 \leq i \leq m_{0}-1$, we denote by $M_{i}=M_{i-1} \backslash\left\{u_{i}\right\}$ the $i^{\text {th }}$ group, and by $m_{i}=\left|M_{i}\right|$ its size. Thus, starting from the initial connection structure $T_{0}$ for $M_{0}$, at each stage of withdrawal $i$, the member $u_{i}$ is removed by updating the current structure $T_{i-1}$ (spanning $M_{i-1}$ ) to obtain $T_{i}$ spanning $M_{i}$.

Note that as the members to remove are revealed one by one, we are in an on-line model. It means that we do not know the future: Neither in which order the members are removed, nor what is the set of members to remove. Hence, each stage can potentially be the last one; this explains why we are interested by giving guarantees at each stage.

We need the following definition that presents the best possible connection tree for the group $M_{k}$, minimizing the diameter parameter.

Definition 2 (Optimal tree). Let $G=(V, E, w)$ be a graph. For every $i$, $0 \leq i \leq m_{0}-1$, we denote by $T_{i}^{*}$ a tree satisfying

$$
D_{T_{i}^{*}}\left(M_{i}\right)=\min \left\{D_{T}\left(M_{i}\right): T \text { tree spanning } M_{i}\right\} \text {. }
$$

We are now ready to give the two constraints that each current structure $T_{i}$ must satisfy.

- The tree constraint: For every $i, 0 \leq i \leq m_{0}-1, T_{i}$ must be a tree, spanning $M_{i}$, in which all leaves are in $M_{i}$ (we call that a pruned tree).

- The quality constraint: Let $c \geq 1$ be any fixed constant representing the required level of quality. For every $i, 0 \leq i \leq m_{0}-1$, we must have $D_{T_{i}}\left(M_{i}\right) \leq c \cdot D_{T_{i}^{*}}\left(M_{i}\right)$. 
As in a net-meeting the current structure $T_{i}$ is used to support the communications between members of $M_{i}$, the tree constraint is set in order to simplify the mechanisms of routing and duplication of information in $T_{i}$. Indeed, there is only one route between any pair of members in a tree; moreover as there is no cycle, a simple flooding process can be used to broadcast information from any member. This flooding naturally ends at the leaves that are members (because trees are pruned); there is no need of costly process to control it.

The quality constraint of level $c$ is set to guarantee that the induced diameter of the current group in $T_{i}$ is not too large compared to the best possible diameter in $T_{i}^{*}$ (at most $c$ times the best possible diameter).

In the rest of the paper we say that an algorithm solves our problem if, for any on-line sequence of successive groups $M_{0} \supset \cdots \supset M_{i}$, it returns a sequence of trees $T_{0}, \ldots, T_{i}\left(T_{i}\right.$ spanning $\left.M_{i}\right)$ satisfying the tree and quality constraints.

\subsection{The criterion to minimize}

In this subsection we present the cost associated with any algorithm satisfying the tree and quality constraints. We first need the following definitions.

Definition 3 (Critical stage). Let $\mathscr{A}$ be an algorithm solving our problem. At stage $i, 1 \leq i \leq m_{0}-1$, Algorithm $\mathscr{A}$ builds $T_{i}=\left(V_{i}, E_{i}\right)$ from $T_{i-1}=$ $\left(V_{i-1}, E_{i-1}\right)$. Stage $i$ is a critical stage if $E_{i} \nsubseteq E_{i-1}$.

We distinguish critical stages from other stages since they generate a lot of perturbations. Indeed, if $i$ is a critical stage, the communication routes in $T_{i-1}$ between members already in the current group $M_{i-1}$ have to be changed in $T_{i}$. Potentially all the routing tables of the connecting nodes must be modified. This generates a heavy traffic to update them. Moreover the current communications between members of $M_{i-1}$ initiated before the changes may be interrupted. That is why the number of critical stages must be minimized.

On the other hand, the withdrawal of a member by just removing useless branches in the tree generates only local changes and is not considered as a critical stage (since in this case $E_{i} \subseteq E_{i-1}$ ). The update of the routing can just be done by broadcasting the information of the departure of the leaving member in the new tree $T_{i}$. This does not create any re-routing between the other members. The aim of this paper is to minimize the total number $\sharp C S\left(T_{0}, \ldots, T_{i}\right)$ of critical stages while respecting the tree and the quality constraints.

\section{Our algorithm CS}

\subsection{Definition of Algorithm CS (Critical Stages)}

To define Algorithm CS, we need the following algorithm, called MD for Minimum Distance. We denote by $\operatorname{MD}(M)$ Algorithm MD applied to group $M$ of size $m$ to find a particular group $M\left(r^{*}\right)$ of size $\left\lfloor\frac{m}{2}\right\rfloor+1$ and what we call its associated root. 


\section{Algorithm $\mathrm{MD}(M)$}

1. For each $r \in M$, sort the $m$ vertices of $M$ by non decreasing value of their distance to $r$ : $r, u_{1}^{r}, \ldots, u_{m-1}^{r}\left(d_{G}\left(r, u_{1}^{r}\right) \leq d_{G}\left(r, u_{2}^{r}\right) \leq \cdots \leq d_{G}\left(r, u_{m-1}^{r}\right)\right)$. Let $M(r)=\left\{r, u_{1}^{r}, \ldots, u_{\left\lfloor\frac{m}{2}\right\rfloor}^{r}\right\}$.

2. Return $r^{*}$ and its associated group $M\left(r^{*}\right)$ such that

$$
d_{G}\left(r^{*}, u_{\left\lfloor\frac{m}{2}\right\rfloor}^{r^{*}}\right)=\min \left\{d_{G}\left(r, u_{\left\lfloor\frac{m}{2}\right\rfloor}^{r}\right): r \in M\right\}
$$

Note that for all $r \in M$, the vertices $u_{1}^{r}, \ldots, u_{m-1}^{r}$ can be sorted by non decreasing value of $d_{G}\left(r, u_{k}^{r}\right)$ and the associated group $M(r)$ can be constructed in polynomial time by using Dijkstra's algorithm. Thus, Algorithm $\operatorname{MD}(M)$ finds $M\left(r^{*}\right)$ and its associated root $r^{*}$ in polynomial time.

The main idea of Algorithm CS is to define particular stages numbers, called rebuilding stages during which we (totally) reconstruct the current tree (to match the quality constraint). Between two successive rebuilding stages, a member is leaving by just removing the dead branches of the current tree (in order to maintain at each stage a pruned tree to satisfy the tree constraint).

The following sequence $\left(a_{k}\right)$ defines the rebuilding stages of our algorithm: $m_{a_{0}}=m_{0}$ is the size of the initial group $M_{0}$ and for every $a_{k}(k \geq 1)$, $m_{a_{k}}=\left\lfloor\frac{m_{a_{k-1}}}{2}\right\rfloor$ is the size of the group $M_{a_{k}}$.

\section{Algorithm CS}

- Initially, at stage $a_{0}=0$ :

CS builds a shortest path tree spanning the first group $M_{0}$, rooted in $r_{0} \in M_{0}$, where $r_{0} \in M_{0}$ is the root found by $\operatorname{MD}\left(M_{0}\right)$.

- After the last rebuilding stage $a_{k}$ :

Let $M_{a_{k}+j}$ be the current group and let $u_{a_{k}+j}$ be the $j^{\text {th }}$ member revealed to be removed since the last rebuilding stage $a_{k}$.

- If $m_{a_{k}+j}>\left\lfloor\frac{m_{a_{k}}}{2}\right\rfloor$ (corresponding to $j<m_{a_{k}}-\left\lfloor\frac{m_{a_{k}}}{2}\right\rfloor$ ): Update the tree $T_{a_{k}+j-1}=\left(V_{a_{k}+j-1}, E_{a_{k}+j-1}\right)$ by pruning potential useless branches.

We obtain the pruned tree $T_{a_{k}+j}=\left(V_{a_{k}+j}, E_{a_{k}+j}\right)$ spanning $M_{a_{k}+j}$ satisfying $E_{a_{k}+j} \subseteq E_{a_{k}+j-1}$.

- Otherwise, we have $m_{a_{k}+j}=\left\lfloor\frac{m_{a_{k}}}{2}\right\rfloor$

(corresponding to $j=m_{a_{k}}-\left\lfloor\frac{m_{a_{k}}}{2}\right\rfloor$ ):

This is a rebuilding stage and we have $m_{a_{k}+j}=\left\lfloor\frac{m_{a_{k}}}{2}\right\rfloor=m_{a_{k+1}}$. Break the current tree and construct $T_{a_{k+1}}$, a shortest path tree spanning $M_{a_{k+1}}$, rooted in $r_{a_{k+1}}$ (where $r_{a_{k+1}} \in M_{a_{k+1}}$ is the root found by $\left.\operatorname{MD}\left(M_{a_{k+1}}\right)\right)$. Thus, $a_{k+1}$ is the new last rebuilding stage. 
The rebuilding stages of CS can be critical stages (because the current tree is broken and rebuilt). The other stages are non critical because the algorithm only removes from the current tree useless branches to obtain the new tree.

Note that this algorithm is polynomial because it uses Algorithm MD (MD is polynomial) and because updating a tree by removing useless branches can be done in polynomial time.

Note also that by construction, at each stage, the tree constraint is satisfied. Section 2.2 shows that it also respects the quality constraint for a level of quality $c=4$.

\subsection{CS respects the quality constraint}

Theorem 1 shows that CS respects the quality constraint with a level of quality $c=4$.

Theorem 1. Let $G=(V, E, w)$ be a graph. For any sequence of withdrawals, at every stage $i, 0 \leq i \leq m_{0}-1$ ( $i$ is the number of removed members), let $T_{i}^{*}$ be an optimal (off-line) tree spanning $M_{i}$ for the diameter. CS respects the quality constraint with a level of quality $c=4$, i.e. for every $i, 0 \leq i \leq m_{0}-1$, we have

Proof.

$$
D_{T_{i}}\left(M_{i}\right) \leq 4 D_{T_{i}^{*}}\left(M_{i}\right)
$$

- If $i$ is a stage of rebuilding. In this case, $i=a_{k}$. Let $u_{0}, v_{0} \in M_{a_{k}}$ be such that $d_{T_{a_{k}}}\left(u_{0}, v_{0}\right)=D_{T_{a_{k}}}\left(M_{a_{k}}\right)$ (where $T_{a_{k}}$ is the tree spanning $M_{a_{k}}$ rooted in $r^{*}$ built by CS at stage $\left.a_{k}\right)$. We have

$$
\begin{aligned}
D_{T_{a_{k}}}\left(M_{a_{k}}\right)= & d_{T_{a_{k}}}\left(u_{0}, v_{0}\right) \leq d_{T_{a_{k}}}\left(u_{0}, r^{*}\right)+d_{T_{a_{k}}}\left(r^{*}, v_{0}\right) \\
& \text { (by triangular inequality) } \\
= & d_{G}\left(u_{0}, r^{*}\right)+d_{G}\left(r^{*}, v_{0}\right) \leq 2 D_{G}\left(M_{a_{k}}\right) \\
& \text { (because } T_{a_{k}} \text { is a shortest path tree rooted } \\
& \text { in } \left.r^{*} \text { and because } u_{0}, v_{0}, r^{*} \in M_{a_{k}}\right) \\
\leq & 2 D_{T_{a_{k}}^{*}}\left(M_{a_{k}}\right) \leq 4 D_{T_{a_{k}}^{*}}\left(M_{a_{k}}\right)
\end{aligned}
$$

(because for every tree $T$ spanning

a group $\left.M, D_{G}(M) \leq D_{T}(M)\right)$

- Otherwise $a_{k}$ is not a stage of rebuilding. Let $j, 1 \leq j<m_{a_{k}}-\left\lfloor\frac{m_{a_{k}}}{2}\right\rfloor$ be the number of removed vertices after the last rebuilding, happening at stage $a_{k}$ (i.e. $j$ is such that $\left.m_{a_{k}+j} \geq\left\lfloor\frac{m_{a_{k}}}{2}\right\rfloor+1\right)$. Let $M\left(r^{*}\right)=\left\{r^{*}, u_{1}^{r^{*}}, \ldots, u_{\left\lfloor\frac{m_{a_{k}}}{2}\right\rfloor}^{r^{*}}\right\}$ be the set returned by $\operatorname{MD}\left(M_{a_{k}}\right)$. As $M_{a_{k}+j} \subset M_{a_{k}}$ (by definition of the sequence of withdrawals) and $M\left(r^{*}\right) \subseteq M_{a_{k}}$ with $m_{a_{k}+j} \geq\left\lfloor\frac{m_{a_{k}}}{2}\right\rfloor+1$ and $\left|M\left(r^{*}\right)\right|=\left\lfloor\frac{m_{a_{k}}}{2}\right\rfloor+1$, we have $M_{a_{k}+j} \cap M\left(r^{*}\right) \neq \emptyset$. Thus, there exists $v \in M_{a_{k}+j} \cap M\left(r^{*}\right)$. As $v \in M\left(r^{*}\right), v=r^{*}$ or $v=u_{l}^{r^{*}}$, with $l \leq\left\lfloor\frac{m_{a_{k}}}{2}\right\rfloor$. As $r^{*}, u_{1}^{r^{*}}, \ldots, u_{\left\lfloor\frac{m_{a_{k}}}{2}\right\rfloor}^{r^{*}}$ are sorted by non decreasing value of their distance to 
$r^{*}$ (see definition of Algorithm MD), we have

$$
d_{G}\left(r^{*}, v\right) \leq d_{G}\left(r^{*}, u_{\left\lfloor\frac{m_{a_{k}}}{2}\right\rfloor}^{r^{*}}\right)
$$

Moreover, as Algorithm $\operatorname{MD}\left(M_{a_{k}}\right)$ finds $r^{*}$ and $M\left(r^{*}\right)$ such that $d_{G}\left(r^{*}, u_{\left\lfloor\frac{m_{a_{k}}}{2}\right\rfloor}^{r^{*}}\right)=\min \left\{d_{G}\left(r, u_{\left\lfloor\frac{m_{a_{k}}}{2}\right\rfloor}^{r}\right): r \in M_{a_{k}}\right\}$, for every $r^{0} \in M_{a_{k}+j} \subset M_{a_{k}}$ we have

$$
d_{G}\left(r^{*}, u_{\left\lfloor\frac{m_{a_{k}}}{2}\right\rfloor}^{r^{*}}\right) \leq d_{G}\left(r^{0}, u_{\left\lfloor\frac{m_{a_{k}}}{2}\right\rfloor}^{r^{0}}\right)
$$

As $m_{a_{k}+j} \geq\left\lfloor\frac{m_{a_{k}}}{2}\right\rfloor+1$ and as $r^{0}, u_{1}^{r^{0}}, \ldots, u_{m_{a_{k}}-1}^{r^{0}}$ are sorted by non decreasing value of their distance to $r^{0}$, there exists $u_{l}^{r^{0}} \in M_{a_{k}+j}$ with $\left\lfloor\frac{m_{a_{k}}}{2}\right\rfloor \leq l \leq$ $m_{a_{k}+j}-1$ such that

$$
d_{G}\left(r^{0}, u_{\left\lfloor\frac{m_{a_{k}}}{2}\right\rfloor}^{r^{0}}\right) \leq d_{G}\left(r^{0}, u_{l}^{r^{0}}\right)
$$

By (1), (2), (3) and as $r^{0}$ and $u_{l}^{r^{0}}$ are in $M_{a_{k}+j}$, by definition of the diameter, we obtain

$$
\exists v \in M_{a_{k}+j} \cap M\left(r^{*}\right): d_{G}\left(r^{*}, v\right) \leq D_{G}\left(M_{a_{k}+j}\right)
$$

Let $u^{0} \in M_{a_{k}+j}$ and $v^{0} \in M_{a_{k}+j}$ be such that $d_{T_{a_{k}+j}}\left(u^{0}, v^{0}\right)=D_{T_{a_{k}+j}}\left(M_{a_{k}+j}\right)$ (where $T_{a_{k}+j}$ is the tree spanning $M_{a_{k}+j}$ built by CS at stage $a_{k}+j$ ). We have

$$
\begin{aligned}
D_{T_{a_{k}+j}}\left(M_{a_{k}+j}\right)= & d_{T_{a_{k}+j}}\left(u^{0}, v^{0}\right)=d_{T_{a_{k}}}\left(u^{0}, v^{0}\right) \\
& (\text { since, by definition of Algorithm CS, } \\
& \text { we have } \left.T_{a_{k}+j} \subseteq T_{a_{k}}\right) \\
\leq & d_{T_{a_{k}}}\left(u^{0}, r^{*}\right)+d_{T_{a_{k}}}\left(r^{*}, v^{0}\right) \\
& (\text { by triangular inequality) } \\
= & d_{G}\left(u^{0}, r^{*}\right)+d_{G}\left(r^{*}, v^{0}\right) \\
& \left(\text { because } T_{a_{k}} \text { is a shortest path tree rooted in } r^{*}\right) \\
\leq & d_{G}\left(u^{0}, v\right)+d_{G}\left(v, r^{*}\right)+d_{G}\left(r^{*}, v\right)+d_{G}\left(v, v^{0}\right) \\
& (\text { by triangular inequality, using vertex } v \text { of }(4)) \\
\leq & 4 D_{G}\left(M_{a_{k}+j}\right) \\
& \left(\text { because } v \in M_{a_{k}+j}, u^{0} \in M_{a_{k}+j},\right. \\
& \left.v^{0} \in M_{a_{k}+j} \text { and by }(4)\right) \\
\leq & 4 D_{T_{a_{k}+j}^{*}}\left(M_{a_{k}+j}\right) \\
& (\text { because for every tree } T \text { spanning } \\
& \text { a group } \left.M, D_{G}(M) \leq D_{T}(M)\right)
\end{aligned}
$$

In conclusion, for every $i, 0 \leq i \leq m_{0}-1$, we obtain $D_{T_{i}}\left(M_{i}\right) \leq 4 D_{T_{i}^{*}}\left(M_{i}\right)$. 


\subsection{CS leads to $O(\log i)$ critical stages}

Theorem 2. Let $G=(V, E, w)$ be a graph. For any sequence of withdrawals, let $T_{0}, \ldots, T_{i}\left(0 \leq i \leq m_{0}-1\right)$ be the sequence of trees constructed by CS. We have

$$
\sharp C S\left(T_{0}, \ldots, T_{i}\right) \leq\left\lfloor\log _{2}(2 i)\right\rfloor=O(\log i)
$$

Proof. Two cases may occur:

- If $i<m_{0}-\left\lfloor\frac{m_{0}}{2}\right\rfloor$, by definition of CS, there is no rebuilding stage. Thus, $C S\left(T_{0}, \ldots, T_{i}\right)=0$.

- Otherwise, $i \geq m_{0}-\left\lfloor\frac{m_{0}}{2}\right\rfloor \geq \frac{m_{0}}{2}$ and we obtain

$$
m_{0} \leq 2 i
$$

Moreover, by definition of the sequence $\left(a_{k}\right)$ and CS, if there are $p$ rebuildings (that are critical stages), then $p$ is such that

$$
\begin{array}{rlrl}
m_{a_{p+1}}<m_{0}-i \leq m_{a_{p}} & \Rightarrow m_{0}-i \leq \frac{m_{0}}{2^{p}} & & \text { (by definiton of sequence }\left(a_{k}\right), \\
& \left.\Rightarrow k, m_{a_{k}} \leq \frac{m_{0}}{2^{k}}\right) \\
& \Rightarrow m_{0}-i \leq \frac{2 i}{2^{p}} & (\text { by }(5)) \\
& \left.\Rightarrow p \leq \frac{2 i}{2^{p}} \quad \quad \text { (by definition, } i \leq m_{0}-1\right) \\
& \Rightarrow p \leq O(\log 2) &
\end{array}
$$

\section{Lower bound for the number of critical stages of any algorithm}

In this section, we prove that for any algorithm respecting the tree constraint and the quality constraint, for any sufficiently large $i$, there exists a particular sequence of withdrawals leading to at least $\Omega(\log i)$ critical stages. To prove that, we describe the graph $G$ in Section 3.1. Then, we define the particular on-line sequence of withdrawals in Section 3.2 and prove the main result in Section 3.3.

\subsection{Description of the graph $G$}

Let $k, d, 0 \leq k \leq d$ and $3 \leq p$ be any integer. We define graphs $G_{k}^{p}=\left(V_{k}^{p}, E_{k}^{p}, w_{k}^{p}\right)$ recursively on $k$ as follows:

- $G_{0}^{p}=\left(V_{0}^{p}, E_{0}^{p}, w_{0}^{p}\right)$ is the cycle of length $p$ such that $\forall e \in E_{0}^{p}, w_{0}^{p}(e)=2^{d}$.

- $\forall k, 1 \leq k \leq d$, we define $G_{k}^{p}=\left(V_{k}^{p}, E_{k}^{p}, w_{k}^{p}\right)$ as follows. $\forall v \in V_{k-1}^{p}$, let $C_{v}=$ $\left(V_{v}^{C}, E_{v}^{C}, w_{v}^{C}\right)$ be a cycle of length $p$ such that $v \in V_{v}^{C},\left(V_{v}^{C} \backslash\{v\}\right) \cap V_{k-1}^{p}=\emptyset$ and $w_{v}^{C}(e)=\frac{2^{d-k}}{p^{k}}$. 
$G_{k}^{p}=\left(V_{k}^{p}, E_{k}^{p}, w_{k}^{p}\right)$ is the graph such that:

- $V_{k}^{p}=V_{k-1}^{p} \cup \bigcup_{v \in V_{k-1}^{p}} V_{v}^{C}$

- $E_{k}^{p}=E_{k-1}^{p} \cup \bigcup_{v \in V_{k-1}^{p}} E_{v}^{C}$

- $\forall e \in E_{k-1}^{p}, w_{k}^{p}(e)=w_{k-1}^{p}(e)$ and $\forall e \in \bigcup_{v \in V_{k-1}^{p}} E_{v}^{C}, w_{k}^{p}(e)=w_{v}^{C}(e)$.

See Figure 1 for an illustration of $G_{k}^{p}$ (with $k=2$ and $p=4$ ). We can now

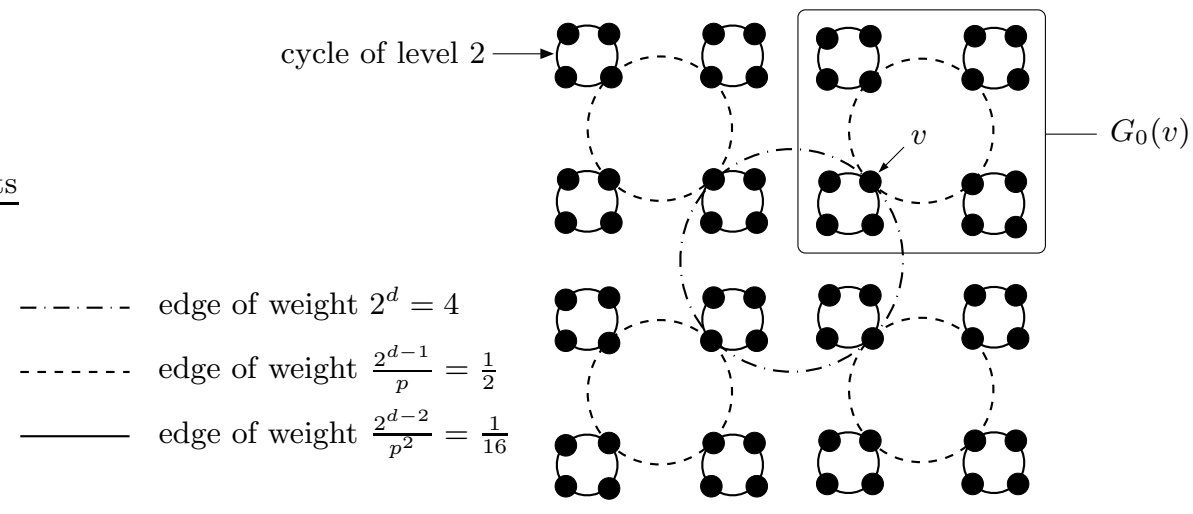

Fig. 1. The graph $G_{2}^{4}$

define the graph $G=(V, E, w)$. Let $c \geq 1$ be the constant corresponding to the required level of quality and let $d$ be a positive integer sufficiently large such that $i \leq\left|V_{d}^{\lceil 6 c+2\rceil}\right|-1$ (where $i$ is the number of removed vertices and $V_{d}^{\lceil 6 c+2\rceil}=M_{0}$ is the initial group). We set $G=G_{d}^{\lceil 6 c+2\rceil}$.

\section{Definition of a cycle of level $k$.}

We say that a cycle $C=\left(V^{C}, E^{C}, w\right)$ (subgraph of $\left.G\right)$ is of level $k(0 \leq k \leq d)$ if each edge $e \in E^{C}$ has weight $w(e)=\frac{2^{d-k}}{p^{k}}$. See Figure 1 for an illustration of such cycle (Note that $G_{2}^{4}$ is too small to be a possible graph of the form $G_{d}^{\lceil 6 c+2\rceil}$, but this is just an illustration).

Definition of the subgraphs $G_{k}(v)$.

Let $v$ be any vertex of the graph $G(v \in V)$. Let $k$ be the smallest index such that $C_{k}=\left(V_{k}^{C}, E_{k}^{C}, w\right)$ is the cycle of level $k$ containing $v\left(v \in V_{k}^{C}\right)$. We define $G_{k}(v)=\left(V_{k}(v), E_{k}(v), w\right)$ the subgraph induced by every vertices and edges which can be reached from vertex $v$ by going through edges of weight strictly less than $\frac{2^{d-k}}{p^{k}}$ (i.e. by going through edges of cycles of level strictly more than $k)$. See Figure 1 for an illustration of such subgraph. 


\subsection{Definition of the sequence of withdrawals $M_{0} \supset \cdots \supset M_{i}$}

Let $A$ be any online algorithm respecting the tree and quality of level $c$ constraints. We use an adaptive adversary to define the sequence of withdrawals in the graph $G=(V, E, w)$ defined above.

We first define a generic sequence of withdrawals of vertices. Note that we do not specify each elementary stage of withdrawal, but only the "main" stages interesting for our analysis (stages of the form $i=\alpha(k, b)$ ). For every $k \geq 0$, for every $b \in\{0,1\}$, for every $i=\alpha(k, b)\left(0 \leq \alpha(k, b) \leq m_{0}-1\right)$, let $T_{i}$ be the tree spanning $M_{i}$ constructed by Algorithm $A$ at stage $i$. Note that at each stage, we have $\alpha(k, b)=\left|M_{0}\right|-\left|M_{\alpha(k, b)}\right|\left(0 \leq \alpha(k, b) \leq m_{0}-1\right)$. The sequence of withdrawals is defined as follows. We set $p=\lceil 6 c+2\rceil$.

\section{Basic Cases:}

- At stage $\alpha(0,0)=0$, we have

$$
M_{\alpha(0,0)}=V
$$

As $T_{\alpha(0,0)}$ is a tree spanning $M_{\alpha(0,0)}$, it is necessarily made up of, amongst other things, all the edges of the cycle $C_{0}=\left(V_{0}^{C}, E_{0}^{C}, w\right)$, except one edge $e_{0}$. Let $v_{0}^{1}$ and $v_{0}^{2}$ be the two vertices connected by $e_{0}$. The adaptive adversary now removes (one by one) from $M_{\alpha(0,0)}$ all the vertices in $\bigcup_{v \in V_{0}^{C} \backslash\left\{v_{0}^{1}, v_{0}^{2}\right\}} V_{1}(v)$ in order to obtain $M_{\alpha(0,1)}$.

- At stage $\alpha(0,1)$, we have

$$
M_{\alpha(0,1)}=V_{1}\left(v_{0}^{1}\right) \cup V_{1}\left(v_{0}^{2}\right)
$$

The adaptive adversary now removes (one by one) from $M_{\alpha(0,1)}$ all the vertices in $V_{1}\left(v_{0}^{1}\right)$ in order to obtain $M_{\alpha(1,0)}$ (note that the adversary chooses arbitrarily to remove all the vertices in $V_{1}\left(v_{0}^{1}\right)$ rather than in $\left.V_{1}\left(v_{0}^{2}\right)\right)$.

\section{Main Cases:}

- At stage $\alpha(k, 0)$. Let $C_{k}=\left(V_{k}^{C}, E_{k}^{C}, w\right)$ be the cycle of level $k$ such that $V_{k}^{C} \subset M_{\alpha(k-1,1)}$. We have

$$
M_{\alpha(k, 0)}=\bigcup_{v \in V_{k}^{C}} V_{k+1}(v)
$$

As $T_{\alpha(k, 0)}$ is a tree spanning $M_{\alpha(k, 0)}$, it is necessarily made up of, amongst other things, all the edges of the cycle $C_{k}$, except one edge $e_{k}$. Let $v_{k}^{1}$ and $v_{k}^{2}$ be the two vertices connected by $e_{k}$. The adaptive adversary now removes (one by one) from $M_{\alpha(k, 0)}$ all the vertices in $\bigcup_{v \in V_{k}^{C} \backslash\left\{v_{k}^{1}, v_{k}^{2}\right\}} V_{k+1}(v)$ in order to obtain $M_{\alpha(k, 1)}$. 
- At stage $\alpha(k, 1)$, we have

$$
M_{\alpha(k, 1)}=V_{k+1}\left(v_{k}^{1}\right) \cup V_{k+1}\left(v_{k}^{2}\right)
$$

The adaptive adversary now removes (one by one) from $M_{\alpha(k, 1)}$ all the vertices in $V_{k+1}\left(v_{k}^{1}\right)$ in order to obtain $M_{\alpha(k+1,0)}$ (note that the adversary chooses arbitrarily to remove all the vertices in $V_{k+1}\left(v_{k}^{1}\right)$ rather than in $\left.V_{k+1}\left(v_{k}^{2}\right)\right)$.

We specify with $\alpha(k, b)$ only the "main" stages of the sequence of withdrawals, corresponding to the stages where the adaptive adversary has to make a choice. Indeed, between two successive "main" stages $\alpha(k, 0)$ and $\alpha(k, 1)$ (resp. $\alpha(k, 1)$ and $\alpha(k+1,0))$, the vertices are removed one by one in any order. Note that we stop removing vertices after the last "main" stage, when exactly $i$ vertices have been removed. See Figure 2 for an illustration of the six first "main" stages $\alpha(0,0), \alpha(0,1), \alpha(1,0), \alpha(1,1), \alpha(2,0)$ and $\alpha(2,1)$, where the successive trees are built by an arbitrary algorithm (Note that $G_{2}^{4}$ is too small to be a possible graph of the form $G_{d}^{\lceil 6 c+2\rceil}$, but this figure is just an illustration of a sequence of withdrawals).
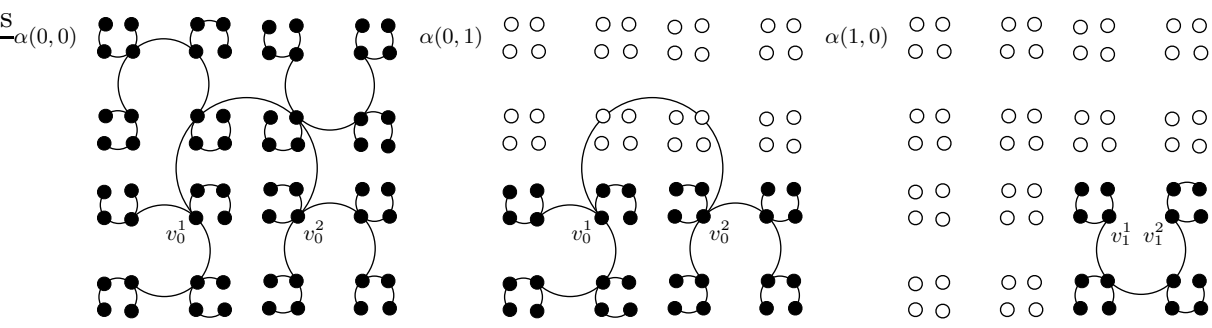

\begin{tabular}{|c|c|c|c|c|c|c|c|c|c|c|c|c|c|c|}
\hline$\alpha(1,1)$ & $\begin{array}{ll}0 & 0 \\
0 & 0\end{array}$ & $\begin{array}{ll}\circ & 0 \\
\circ & 0\end{array}$ & $\begin{array}{ll}\circ & 0 \\
0 & 0\end{array}$ & $\begin{array}{ll}\circ & 0 \\
0 & 0\end{array}$ & $\alpha(2,0)$ & $\begin{array}{ll}\circ & 0 \\
\circ & 0\end{array}$ & $\begin{array}{ll}\circ & 0 \\
\circ & 0\end{array}$ & $\begin{array}{ll}\circ & 0 \\
0 & 0\end{array}$ & $\begin{array}{ll}\circ & 0 \\
\circ & 0\end{array}$ & $\alpha(2,1)$ & $\begin{array}{ll}\circ & 0 \\
\circ & 0\end{array}$ & $\begin{array}{ll}\circ & 0 \\
\circ & 0\end{array}$ & $\begin{array}{ll}\circ & 0 \\
\circ & 0\end{array}$ & $\begin{array}{ll}\circ & 0 \\
\circ & 0\end{array}$ \\
\hline & ○ 0 & ○ 0 & ० & 00 & & 00 & 00 & 00 & $\circ \circ$ & & 00 & 00 & 00 & \\
\hline & 00 & 00 & 00 & 00 & & 00 & 00 & 00 & 00 & & $\circ 0$ & 00 & 00 & \\
\hline & 00 & ○ 0 & & & & 00 & 00 & 8 & 00 & & 00 & O O & oro & \\
\hline & 00 & 00 & & & & 00 & 00 & $v_{2}^{1} v_{2}^{2}$ & 00 & & 00 & 00 & ${ }_{v_{2}^{1}} v_{2}^{2}$ & \\
\hline & 00 & 00 & Or & o & & 00 & 00 & 00 & 00 & & 00 & 00 & 00 & \\
\hline & 00 & 00 & 00 & 00 & & 00 & 00 & 00 & 00 & & 00 & 00 & 00 & \\
\hline
\end{tabular}

Fig. 2. Illustration of the sequence of withdrawals on graph $G_{2}^{4}$

\subsection{Any algorithm leads to $\Omega(\log i)$ critical stages}

Lemmas 1 and 2 are preliminary technical results (Lemma 1 is trivial. A proof can be found in [5]). 
Lemma 1 Let $G=(V, E, w)$ be any graph. For every $M \subseteq V$, there exists a tree $T^{\mathrm{off}}$ spanning $M$ such that

$$
D_{T^{\text {off }}}(M) \leq 2 D_{G}(M)
$$

The following Lemma is central in our analysis. It describes sub-sequences of withdrawals where at least one rebuilding/critical stage occurs.

Lemma 2 Let $c \geq 1$ be any constant (representing the required level of quality). For every $k \geq 0$, let $T_{\alpha(k, 0)}^{*}, T_{\alpha(k, 0)+1}^{*}, \ldots, T_{\alpha(k, 1)}^{*}$ be the trees respectively spanning $M_{\alpha(k, 0)}, M_{\alpha(k, 0)+1}, \ldots, M_{\alpha(k, 1)}$ optimal for the diameter and let $T_{\alpha(k, 0)}$, $T_{\alpha(k, 0)+1}, \ldots, T_{\alpha(k, 1)}$ be any trees respectively spanning $M_{\alpha(k, 0)}, M_{\alpha(k, 0)+1}, \ldots$, $M_{\alpha(k, 1)}$. If for every $i, \alpha(k, 0) \leq i \leq \alpha(k, 1)$, we have $D_{T_{i}}\left(M_{i}\right) \leq c \cdot D_{T_{i}^{*}}\left(M_{i}\right)$, then

$$
\sharp C S\left(T_{\alpha(k, 0)}, T_{\alpha(k, 0)+1}, \ldots, T_{\alpha(k, 1)}\right) \geq 1 .
$$

Proof. We prove Lemma 2 by contradiction. Suppose that there exists $k \geq 0$ such that for every $i, \alpha(k, 0) \leq i \leq \alpha(k, 1)$, the quality constraint is satisfied and there is no critical stage, i.e. there exists $k \geq 0$ such that for every $i, \alpha(k, 0) \leq i \leq$ $\alpha(k, 1)$, we have $D_{T_{i}}\left(M_{i}\right) \leq c \cdot D_{T_{i}^{*}}\left(M_{i}\right)$ and $T_{\alpha(k, 0)} \supseteq T_{\alpha(k, 0)+1} \supseteq \cdots \supseteq T_{\alpha(k, 1)}$.

These trees are made up of, amongst other things, all edges of the cycle $C_{k} \subset$ $G$, except one edge, noted $e_{k}$. We insist on the fact that, because there is no critical stage, this edge $e_{k}$ is always the same in all trees $T_{\alpha(k, 0)}, T_{\alpha(k, 0)+1}, \ldots, T_{\alpha(k, 1)}$.

Let us focus now on stage $\alpha(k, 1)$, where $M_{\alpha(k, 1)}=V_{k+1}\left(v_{k}^{1}\right) \cup V_{k+1}\left(v_{k}^{2}\right)$. We lower bound $D_{T_{\alpha(k, 1)}}\left(M_{\alpha(k, 1)}\right)$ and upper bound $D_{T_{\alpha(k, 1)}^{*}}\left(M_{\alpha(k, 1)}\right)$ to show that at this particular stage, the quality constraint is not satisfied. This leads to the wanted contradiction and proves the Lemma.

- Lower bound of $D_{T_{\alpha(k, 1)}}\left(M_{\alpha(k, 1)}\right)$.

As the two extremities $v_{k}^{1}$ and $v_{k}^{2}$ of the edge $e_{k}$ are separated by a path made of $p-1=\lceil 6 c+1\rceil$ edges of weight $\frac{2^{d-k}}{p^{k}}$ in $T_{\alpha(k, 1)}$ we have

$$
D_{T_{\alpha(k, 1)}}\left(M_{\alpha(k, 1)}\right) \geq(p-1) \frac{2^{d-k}}{p^{k}} \geq(6 c+1) \frac{2^{d-k}}{p^{k}}
$$

- Upper bound of $D_{T_{\alpha(k, 1)}^{*}}\left(M_{\alpha(k, 1)}\right)$.

In order to upper bound $D_{T_{\alpha(k, 1)}^{*}}\left(M_{\alpha(k, 1)}\right)$, we first upper bound $D_{G}\left(M_{\alpha(k, 1)}\right)$. By construction of the graph $G$, two cases may occur:

1. If $k=d$, there is no cycle of level $k+1$ in $G$. Thus, we have

$$
D_{G}\left(M_{\alpha(k, 1)}\right)=w\left(e_{k}\right)=\frac{2^{d-k}}{p^{k}} \leq 3 \frac{2^{d-k}}{p^{k}}
$$


2. If $k \leq d-1$, we have

$$
\begin{aligned}
D_{G}\left(M_{\alpha(k, 1)}\right) \leq & D_{G}\left(V_{k+1}\left(v_{k}^{1}\right)\right)+d_{G}\left(v_{k}^{1}, v_{k}^{2}\right)+D_{G}\left(V_{k+1}\left(v_{k}^{2}\right)\right) \\
\leq & \sum_{e \in E_{k+1}\left(v_{k}^{1}\right)} w(e)+w\left(e_{k}\right)+\sum_{e \in E_{k+1}\left(v_{k}^{2}\right)} w(e) \\
& \quad \text { because for every graph or subgraph } \\
& \left.\quad G=(V, E, w), D_{G}(V) \leq \sum_{e \in E} w(e)\right) \\
= & \sum_{l=k+1}^{d} \frac{2^{d-l}}{p^{l}} p^{l-k}+\frac{2^{d-k}}{p^{k}}+\sum_{l=k+1}^{d} \frac{2^{d-l}}{p^{l}} p^{l-k} \\
\leq & \frac{2}{p^{k}} \sum_{l=k+1}^{d} 2^{d-l}+\frac{2^{d-k}}{p^{k}} \leq 2 \frac{2^{d-k}}{p^{k}}+\frac{2^{d-k}}{p^{k}}=3 \frac{2^{d-k}}{p^{k}}
\end{aligned}
$$

Moreover, by Lemma 1 , there exists a tree $T_{\alpha(k, 1)}^{\mathrm{off}}$ spanning $M_{\alpha(k, 1)}$ such that $D_{T_{\alpha(k, 1)}^{\text {off }}}\left(M_{\alpha(k, 1)}\right) \leq 2 D_{G}\left(M_{\alpha(k, 1)}\right)$. Thus, as $T_{\alpha(k, 1)}^{*}$ is a tree spanning $M_{\alpha(k, 1)}$ optimal for the diameter, we have

$$
D_{T_{\alpha(k, 1)}^{*}}\left(M_{\alpha(k, 1)}\right) \leq D_{T_{\alpha(k, 1)}^{\mathrm{off}}}\left(M_{\alpha(k, 1)}\right) \leq 2 D_{G}\left(M_{\alpha(k, 1)}\right) \leq 6 \frac{2^{d-k}}{p^{k}}
$$

By (6) and (7), we obtain

$$
\frac{D_{T_{\alpha(k, 1)}}\left(M_{\alpha(k, 1)}\right)}{D_{T_{\alpha(k, 1)}^{*}}\left(M_{\alpha(k, 1)}\right)} \geq \frac{(6 c+1) \frac{2^{d-k}}{p^{k}}}{6 \frac{2^{d-k}}{p^{k}}} \geq c+\frac{1}{6}>c
$$

This result contradicts the assumption that the quality constraint is satisfied. Thus, Lemma 2 is proved by contradiction.

The following Theorem shows that if the tree constraint and the quality constraint are satisfied, any algorithm leads to $\Omega(\log i)$ critical stages, where $i$ is the number of removed vertices.

Theorem 3. Let $c \geq 1$ be any constant. For any algorithm A, for every sufficiently large $i$, there exists a graph $G_{0}$, there exists $M_{0} \supset \cdots \supset M_{i}$, such that if Algorithm $A$ returns a sequence of trees $T_{0}, \ldots, T_{i}$ respectively spanning $M_{0} \supset \cdots \supset M_{i}$ respecting the quality constraint of level $c$, then

$$
\sharp C S\left(T_{0}, \ldots, T_{i}\right)=\Omega(\log i)
$$

Proof. Let $c \geq 1$ be any constant. We set $p=\lceil 6 c+2\rceil$. Let $i$ be the number of removed vertices. There exists $d$ and $G_{0}$ (where $G_{0}$ is graph $G$, defined in Section 3.1), there exists $M_{0} \supset \cdots \supset M_{i}$ (the sequence defined in Section 3.2) such that

$$
\alpha(d-1,1) \leq i \leq \alpha(d, 1) \leq|V|=p^{d}
$$


Thus, we have $i \leq p^{d} \Rightarrow \log _{p} i \leq d \quad \Rightarrow \quad \log _{p} i \leq d$. And as $p=\lceil 6 c+2\rceil$ is a constant, we have $d \geq \Omega(\log i)$. Moreover, by Lemma 2, we have

$$
\begin{aligned}
& \left\{\begin{array}{c}
\sharp C S\left(T_{\alpha(0,0)}, T_{\alpha(0,0)+1}, \ldots, T_{\alpha(0,1)}\right) \geq 1 \\
\sharp C S\left(T_{\alpha(1,0)}, T_{\alpha(1,0)+1}, \ldots, T_{\alpha(1,1)}\right) \geq 1 \\
\vdots \\
\sharp C S\left(T_{\alpha(d-1,0)}, T_{\alpha(d-1,0)+1}, \ldots, T_{\alpha(d-1,1)}\right) \geq 1
\end{array}\right. \\
& \Rightarrow \sharp C S\left(T_{\alpha(0,0)}, \ldots, T_{\alpha(d-1,1)}\right) \geq d \\
& \left.\Rightarrow \sharp C S\left(T_{0}, \ldots, T_{i}\right) \geq d \quad \text { (because } i \geq \alpha(d-1,1)\right) \\
& \left.\Rightarrow \sharp C S\left(T_{0}, \ldots, T_{i}\right) \geq \Omega(\log i) \quad \text { (because } d \geq \Omega(\log i)\right)
\end{aligned}
$$

Theorem 2 and Theorem 3 show that Algorithm CS is worst case optimal in order of magnitude for the number of critical stages criterion.

\section{Conclusion}

We have proposed an algorithm, called CS, solving an on-line covering problem of members by respecting the following quality constraint: For each stage of withdrawal, the diameter between members induced by the built tree is at most a constant time the best possible value. Moreover, our algorithm is easy to use. Indeed, for a stage of withdrawal, either it breaks the tree and rebuilds a new one which is a tree of shortest paths (only $O(\log i)$ times, where $i$ is the number of removed members), or it just updates the current tree by removing useless branches (in all the other cases).

Moreover, our algorithm is worst case optimal in order of magnitude for the number of critical stages: It leads to $O(\log i)$ critical stages and we showed that any algorithm leads to $\Omega(\log i)$ critical stages in the worst case. We also have proved that the number of elementary changes per stage (see equivalent definition in [4]) is constant in average. Due to space limitation, we do not include these results. Note that we only consider the decremental problem because the incremental problem (adding new members in the current tree) considering the diameter as quality constraint is trivial. Indeed, plugging each new member with a shortest path to the initial member leads to 0 critical stage with a level of quality $c=2$. We also have results with another objective function than the diameter. Indeed, concerning the average distance between members of the groups, we proved similar results in [7] for the incremental version of the problem. We are now currently working on mixing additions and withdrawals.

\section{Acknowledgments}

The authors wish to thank the anonymous referees for their very useful comments. 


\section{References}

1. G. Ausiello, P. Crescenzi, G. Gambosi, V. Kann, A. Marchetti SpaCCamela, and M. Protasi, Complexity and approximation, Springer, 1999.

2. A. Borodin ANd R. El-YAniv, Online computation and competitive analysis, Cambridge University press, 1998.

3. D. HochBaum, Approximation algorithms for NP-hard problems, PWS publishing compagny, 1997

4. M. Imase And B. WaXman, Dynamic steiner tree problem, SIAM J. Discr. Math., 4 (1991), pp. 369-384.

5. C. LAFOREST, A good balance between weight and distances for multipoint trees, in International Conference On Principles Of DIstributed Systems 2002, pp. 195-204.

6. S. Raghavan, G. Manimaran, and C. S. R. Murthy, A rearrangeable algorithm for the construction of delay-constrained dynamic multicast trees, IEEE/ACM (SIGCOMM), ACM Press, 7 (1999).

7. N. ThiBAult And C. LAFOREST, An optimal rebuilding strategy for an incremental tree problem, submitted in 2004 to journal of interconnection networks.

8. B. Waxman, Routing of multipoint connections, IEEE Journal on Selected Areas in Communications, 6 (1988), pp. 1617-1622. 